\title{
Estimación de superficies de cultivos en pequeños regadíos mediante encuesta de terreno y datos de satélite *
}

\author{
MA Casterad 1, M Aran 2, J Herrero **,1, L Albizua 2 \\ 1 SIA-Diputación General de Aragón, BP 727, E50080 Zaragoza; \\ 2 TCSA-Gobierno dé Navarra, Carretera EI Sadar, E 31006 Pamplona, España
}

(Récibido el 5 de agosto de 1991; acceptado el 9 de septiembre de 1992)

\begin{abstract}
Résumé - Estimation des superficies de cultures dans des petits secteurs irrigués par enquête de terrain et données satellitaires. La télédétection est un important outil pour le suivi et l'évaluation des zones agricoles, dont les principaux revenus dans l'Espagne semi-aride viennent des secteurs irrigués. Des problèmes de compétition pour l'eau, d'environnement et de marché rendent nécessaire une information rapide et précise sur l'extension des différentes cultures dans chaque secteur irrigué. La méthode classique d'échantillonnage aréolaire corrigé par l'estimateur de régression, a été adaptée à l'inventaire de cultures dans 3 secteurs irrigués du bassin de l'Ebre (Aragón, Espagne). La petite taille de ces secteurs met la méthode à la limite de son applicabilité, et on a testé l'utilisation de taux d'échantillonnage supérieurs aux taux habituels. Les statistiques de l'utilisation des terres en été sont présentées. Le traitement en ordinateur d'une seule image Landsat TM diminue le coefficient de variation de l'estimation des surfaces de maîs, luzerne et riz, ainsi que des surfaces labourées, avec végétation naturelle ou nues. Dans les cultures minoritaires (tournesol, arbres fruitiers et plantations horticoles) qui sont dispersées et surtout celles qui ont une signature spectrale hétérogène, les estimations sont de mauvaise qualité.
\end{abstract}

télédétection / statistique agricole / échantillonnage aréolaire / méthode de régression / estimation de superficies

Summary - Estimating crop acreage in small irrigated districts via ground-gathered and satellite data. Remote sensing is an important tool for monitoring and evaluating agricultural areas. In the semi-arid part of Spain which are dependent upon irrigation, problems connected with water scarcity, the environment and marketing necessitate the obtention of rapid and accurate crop inventories in each irrigated district. The classical method of area frame sampling corrected by regression estimator has been adapted to the crop acreage in 3 irrigated districts of the central Ebro basin (northeastern Spain). The small size of the districts involved pushes the method to the limits of its applicability; higher ground sampling rates were therefore tested. The statistics of summer land use in these districts have been presented. Computer processing of one single Landsat TM image reduces the coefficient of variation for estimation of the acreages of maize, alfalfa, rice and tilled land (either with natural vegetation or bare soil). The acreage estimation was inaccurate only for minor crops (sunflower, fruit tree and horticultural crops) that are sparsely planted and in particular for those with a heterogeneous spectral signature.

remote sensing / agricultural statistics / area frame sampling / regression analysis / crop acreage

\footnotetext{
* Ese trabajo está realizado con arreglo al proyecto $n^{\circ} 8525$, financiado par INIA

* Correspondencia y tiradas aparte.
}

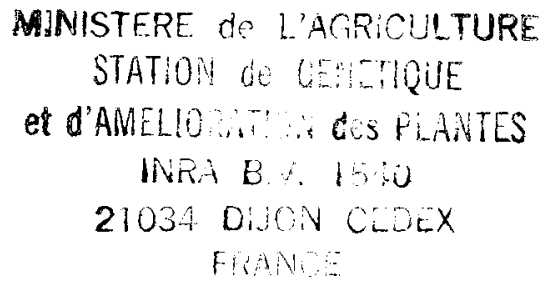




\section{INTRODUCCION}

El clima árido o semiárido del centro de Aragón impone en secano a la cebada como casi único cultivo, con parvas o nulas cosechas en la mayoría de los años. Unas rentas razonablemente seguras para los agricultore aún existentes han sido posibles mediante los regadíos. Sin embargo, en ciertas áreas de Europa como es el caso del valle del Ebro, la política agraria común va a repercutir no sólo sobre los secanos sino incluso sobre los regadíos, que hace pocos años parecian incuestionables. Ello, junto a la creciente escasez de agua y las afecciones medioambientales por salinidad, demanda un detallado seguimiento y control del regadio.

Un dato básico para la gestión y evaluación de un polígono de riego es el conocimiento de la superficie ocupada por cada cultivo, así como de las áreas desnudas y con vegetación espontánea. Además de su valor estadístico, esta información permitirá estimar el grado de maduración del regadio y, si además está georreferenciada, reconocer y localizar problemas de salinidad o limitantes similares.

Las estadísticas tradicionales en muchos países no cubren estas necesidades. Así, en España tales estadísticas desglosan las superficies de cultivos únicamente por municipios, con lo que pocas veces se puede atribuir sin dudas la superficie de un determinado cultivo a un polígono de riego, ni disponer de agregaciones diferentes de sumas de municipios completos.

La teledetección desde satélites representa un avance en ese sentido, y además da las superficies de los cultivos durante la campaña, cuando los datos son más valiosos. La georreferenciación de toda la información y su periodicidad desde hace casi veinte años, son otros alicientes para poner a punto esta herramienta.

Tras las investigaciones iniciades en 1938 por la Universidad de lowa, el Departamento de Agricultura de los Estados Unidos (USDA) realiza estadísticas desde los años 50 mediante encuestas por marco areolar (Cotter y Nealon, 1987). Posteriormente se empezaron a utilizar datos del satélite Landsat para majorar mediante estimador por regresión (Cochran, 1977) las estadísticas obtenidas de las encuestas (Hanuschak et al, 1979, 1982). La misma metodología es aplicada por el organismo canadiense de estadistica (Germain y Julien, 1988).

En Europa, la Comunidad Económica Europea a través del Instituto para las Aplicaciones de la Teledetección del Centro Común de Investigación en Ispra, ha adaptado esta metodología y desarrolla, desde 1988 , un proyecto piloto de diez años de duración en el que se aplica la teledetección a las estadísticas agrarias (Delince, 1988; IAT, 1989; Gallego, 1990; Meyer-Roux, 1990). El plan incluye la obtención de inventarios por regiones y estimaciones rápidas en la CEE mediante la citada metodología.

El método del marco areolar y estimador por regresión con datos de satélite es aplicado desde hace años en Francia por el Ministerio de Agricultura (Meyer-Roux, 1981; Meyer-Roux et al, 1983; Touzelet, 1983; SCEES, 1986). También en Italia (CITA, 1987) y en España se ha aplicado dicha metodología (TCSA, 1987, 1990; SERN, 1990; Gonzalez et al, 1991).

La obtención de estimaciones referidas a pequeñas superficies a partir de la información recogida para grandes áreas, por una parte, y de teledetección por otra, se ha desarrollado en los últimos años (Ford et al, 1983; Battese y Fuller, 1988; Battese et al, 1988; Hultine y Harville, 1991). Sin embargo, no cabe esperar que organismos supranacionales se ocupen de la obtención de estadísticas en pequeñas demarcaciones tales como polígonos de riego, áreas siniestradas 0 a proteger, etc. Estas áreas son a menudo unidades de manejo o de intervención para la administración o para los operadores económicos. La encuesta de marco areolar y estimador de regresión puede aportar un importante avance gracias a la intervención de los datos del satélite.

Estas consideraciones indujeron al Servicio de Investigación Agraria de Aragón a centrarse en el aforo de superficies de cultivo en pequeñas demarcaciones (Casterad, 1990; Gil, 1991; Marinho, 1992; com pers), identificando una serie de problemas estadisticos al ir descendiendo a superficies más y más pequeñas.

El presente trabajo muestra la aplicación del método a tres regadíos aragoneses, con diferentes tasas de muestreo. Se apura en su aplicación, tanto por tratarse de polígonos de riego de dimensiones inferiores a las habituales en trabajos similares, como por haber aforado cultivos cuya distribución y representación están en el límite de la capacidad del método.

\section{AREAS ESTUDIADAS}

Se presentan resultados de tres áreas en las provincias de Zaragoza y Huesca. Sus altitudes 
oscilan entre 200 y $400 \mathrm{~m}$. Las tres áreas son semiáridas, con medias anuales que van desde los $316 \mathrm{~mm}$ de precipitación y $1197 \mathrm{~mm}$ de $E T_{0}$ en el área más al centro del valle del Ebro, hasta los $497 \mathrm{~mm}$ de precipitación y $1054 \mathrm{~mm}$ de $\mathrm{ET}_{0}$ en el área más norteña. Los regímenes de humedad de los suelos son el arídico y el xérico. Los cultivos con mayor consumo unitario de riego son el arroz, la alfalfa, y el maiz.

Las tres áreas son representativas de importantes sistemas agrícolas aragoneses. Junto con ello, el disponer de información de suelos, llevó a elegirlas para aplicar el método de los segmentos al aforo de superficies de cultivos.

La primera de las áreas es la Huerta Vieja del Ebro en los diez municipios ribereños situados aguas abajo del de Zaragoza. Son 15435 ha de regadio tradicional en la llanura de inundación y terrazas inferiores del Ebro. Predominan el maíz, alfalfa, trigo, cebada, frutales y hortícolas, todos con riego a pie. Los suelos presentan sólo ocasionalmente manchas salinas. El parcelario es irregular al coexistir huertos familiares con parcelas resultantes de la concentración. Las aguas de riego proceden del Canal Imperial y de azudes de Ebro.

La segunda de las áreas estudiadas es el Nuevo Regadío de Quinto, que empezó en 1985 y ocupa 3223 ha que se proyectan ampliar. Esta demarcación comprende algunas parcelas excluidas de riego o no amuebladas en el momento del aforo. Los cultivos principales son: cereal de invierno, maíz, alfalfa, guisantes, frutales y girasol. Son frecuentes los suelos yesosos. Además hay inclusiones de suelos salinos que han exigido obras de drenaje. El riego es por aspersión y por goteo; otros datos acerca de este polígono se pueden encontrar en Herrero y Bercero (1991).

La tercera es el área de Flumen, que comprende 25746 ha entre los ríos Flumen y Alcanadre y el canal del Flumen. Casi toda la demarcación está dominada por el canal del Flumen; sólo algunos enclaves permanecen en secano. Además de los sectores 4 al 11 de los regadíos del Flumen, incluye huertas viejas que sólo suponen una pequeña extensión frente a los regadíos de entre 20-50 años de antigüedad. Los cultivos predominantes son maíz, alfalfa, girasol, arroz, trigo y cebada. El riego es casi exclusivamente a pie y las parcelas suelen ser menores de una hectárea. El tipo de suelos y las considerables extensiones afectadas por salinidad (Herrero, 1987; Herrero y Aragüés, 1988; Rodríguez et al, 1989) son también rasgos destacables de este regadío.

\section{MATERIAL Y MÉTODOS}

Se ha analizado la imagen del 20 de julio de 1990 , correspondiente a la escena 199-031 del landsat-5. La información del satélite en cintas magnéticas compatibles con ordenador se trató con un equipo Dipix Aries III.

La referencia cartográfica fueron los mapas a escala 1: 50 000. La información de campo se recogió, en el caso de la Huerta Vieja del Ebro y del Nuevo Regadio de Quinto, sobre ortofotos escala 1: 5000. En Flumen se dispuso de foto aérea de escala 1: 12000 y se emplearon sus ampliaciones a aproximadamente 1: 8000 . Además de la información sobre los cultivos, se reflejaban caracteristicas del territorio de interés en el posterior proceso de la información digital de los cultivos y los suelos.

El método aplicado fue el de encuesta de terreno por muestreo de marco areolar y estimator por regresión con la clasifiación supervisada de la información multiespectral del satélite, basado en los desarrollos estadisticos de Cochran (1977). El método comporta una primera etapa de encuesta de terreno en base a unas unidades de muestreo denominadas segmentos, elegidos al azar.

Una primera estimación de la superficie de cada cultivo para cada regadio se obtiene por expansión, multiplicando la superficie media que ocupa el cultivo en cuestión en los segmentos, por el número de segmentos posibles en ese regadio $(N)$. El análisis de los datos permite estimar la varianza del muestreo y por tanto el error tipico que se traduce en un coeficiente de variación de la estimación

A partir de la experiencia en el valle del Ebro (Casterad, 1990; TCSA, 1990), se han adoptado segmentos de $500 \times 500 \mathrm{~m}$. La tasa de muestreo fue del $3,2 \%$ (20 segmentos) para la ribera del Ebro. En el nuevo regadio de Quinto fue del 14,7\% (19 segmentos) y del $2,5 \%$ (26 segmentos) en Flumen. Los trabajos de campo duraron un mes desde el 18 de julio. La información recogida sirvió para la expansión y para tomar áreas de verdad-terreno en la clasificación.

En una segunda fase, aplicación del estimador por regresión, los datos obtenidos por expansión se corrigen mediante la clasificación supervisada de los datos de satélite. Para ello se procesan las imágenes (comprobación de la calidad de la imagen, corrección geométrica, remuestreo de la información, estratificación del territorio, incorporación de datos de verdadterreno, creación de firmas espectrales y clasificación), atribuyéndose finalmente un número de píxeles a cada una de las clases de cultivos y usos del territorio. Estos pixeles se expresan en hectáreas (Cuadros 1 a 3) para facilitar la comparación. En este estudio se realizó clasificación supervisada por máxima verosimiltud de todas las bandas excepto la seis. 
Tras la clasificación se ajusta, para cada clase temática, la regresión lineal de las superficies de verdad-terreno sobre las obtenidas por clasificación supervisada en los segmentos. Se presentan el coeficiente de determinación $\left(r^{2}\right)$ y el nivel de significación de la regresión. La incorporación de la clasificación multiespectral en las clases cuya regresión resulta significativa mejora el dato obtenido por expansión. La teledetección mejora poco la estimación cuando el coeficiente de correlación es pequeño.

Debe establecerse, para cada clase temática, la superficie media por segmento corregida con la clasificación multiespectral, $\hat{\bar{Y}}$. Este valor, multiplicado por el número de segmentos posibles en todo el territorio, da la superficie por regresión $(\hat{\bar{Y}} M)$. Se utiliza la fórmula (Cochran, 1977):

$$
\hat{\bar{Y}}=\ddot{y}+b(\ddot{X}-\bar{x}), \text { donde : }
$$

$\bar{y}=$ superficie media de la clase en los segmentos, según los datos de verdad-terreno; $b=$ pendiente de la recta de regresión de las superficies de verdadterreno sobre las de clasificación multiespectral en los segmentos; $\bar{x}$ : superficie media de la clase en los segmentos, según los datos de clasificación multiespectral; $\bar{x}=$ superficie media de la clase que correspondería a cada uno de los segmentos posibles en el territorio, según la clasificación multiespectral de todo el territorio.

La fórmula afina la estimación obtenida por muestreo al azar (verdad-terreno), corrigiéndola al incorporar la clasificación multiespectral en el factor $(\bar{X}-\bar{x})$, ponderada a su vez por el grado de correspondencia entre la superficie obtenida por la clasificación según respuesta espectral de una clase temática y la superficie inventariada. Esta ponderación se incorpora a la fórmula mediante el valor (b) de la pendiente de la regresión de la verdad-terreno sobre la clasificación.

Además de la superficie media, se presentan el coeficiente de variación de la estimación por regresión, indicator de la precisión final alcanzada, y la eficiencia relativa del método, $E R=\left(1-r^{2}\right)^{-1}$, que indica cuántas veces habria que aumentar la tasa de muestreo para lograr el mismo coeficiente de variación si se utilizase sólo la expansión.

\section{RESULTADOS Y DISCUSION}

Para la visualización de las imágenes se utilizaron las bandas 4-5-3 (RGB). En ellas se identifjcan los cultivos por su tonalidad y se localizan sus marras. Destacan en rojo las parcelas con vegetación de regadío (alta reflectividad en el infrarrojo próximo), frente a los tonos apagados del secano. Además, esas parcelas muestran tonalidades según las diferentes especies: maíz (rojo), alfalfa (rojo muy vivo) y matices correspondientes a estados fenológicos. La vegetación de ribera aparece rojo-marrón.
El tamaño y la forma de las parcelas son otros aspectos diferenciadores: mosaico de pequeñas parcelas con diversidad de usos en la Huerta Vieja, grandes y de formas más monótonas en el Nuevo Regadío. Las irregularidades intraparcelares en la imagen, son muy marcadas en ciertas parcelas del Nuevo Regadio. Estos rodales se asocian generalmente a marras del cultivo de origen diverso (encharcamiento, salinidad, suelo somero, mala nascencia, etc). En la imagen de Flumen, además del mosaico complejo del parcelario, destaca la variedad de tonos dentro de una misma clase debida a la salinidad que afecta a amplias extensiones.

Se han obtenido las respuestas espectrales de los usos siguientes: agua, áreas urbanas, alfalfa, arroz, barbecho, cebolla, frutales, girasol, maiz, rastrojera, terreno arado, vegetación espontánea, vegetación de ribera. Las superficies de cereal de invierno tanto en secano como en regadío, así como las áreas de barbecho, requeririan una imagen de primavera con trabajo de campo en fechas coincidentes.

Los Cuadros I, II y III presentan solamente los aforos de las superficies de los cultivos más extendidos, ya que el método utilizado no es aplicable a usos de escasa superficie. Es el caso de los frutales, cuya escasez y diversidad en el manejo de las plantaciones impiden una buena selección de áreas de entrenamiento del clasificador.

Tampoco se presentan en los Cuadros los resultados del girasol, cuya pequeña superficie y poca representación en el regadio lo deja fuera del límite de aplicabilidad del método. Por expansión se obtienen 148,4 ha con $\mathrm{CV}$ de $43,0 \%$ en el Nuevo Regadio, y 1513.8 ha con CV de $39,2 \%$ en Flumen. Al aplicar la regresión de verdad-terreno sobre la clasificación, estos resultados no mejoran. Se obtienen valores de $r^{2}$ de 0,14 en el Nuevo Regadio y de 0,10 en Flumen que no son significativos al $95 \%$. Ello se asocia a la heterogeneidad de la firma espectral en esta fecha puesto que hay parcelas en flor, otras en desarrollo y otras en plántula, a causa de las distintas variedades y el escalonamiento de siembras.

Los cultivos presentados más el terreno arado, que corresponde aproximadamente a la superficie de cereal de invierno, suponen el $53,6 \%$ de la Huerta Vieja, el $72,1 \%$ del Nuevo Regadio y el $57,1 \%$ de Flumen. Estas superficies no comprenden los cultivos no aforables ni otras clases no agrícolas. Tales usos del territorio están más 
Cuadro I. Estimación de las superficies ocupadas por los principales usos y cultivos en las 15435 ha de la Huerta Vieja de diez municipios ribereños del Ebro (Zaragoza), Julio 1990.

\begin{tabular}{|c|c|c|c|c|c|c|c|c|}
\hline Clases & $\begin{array}{c}\text { Expansión }(\bar{y} N) \\
\text { (ha) }\end{array}$ & $\begin{array}{l}\text { Clasificación }(\bar{X} N) \\
\quad \text { (pixeles } \\
\text { convertidos a ha) }\end{array}$ & \multicolumn{2}{|c|}{ Regresión $(\tilde{\bar{Y}} N)$} & \multicolumn{2}{|c|}{$\begin{array}{c}C V(\%) \\
\text { Expansión Regresión }\end{array}$} & $r^{2}$ & $E R$ \\
\hline Maiz & 3412.8 & 2224.9 & 2621.8 & 17.0 & 15.9 & 11.4 & $0.70^{\star *}$ & 3.3 \\
\hline Alfalfa & 3053.9 & 2408.1 & 2628.4 & 17.0 & 14.4 & 10.6 & $0.60^{\star \star}$ & 2.5 \\
\hline Rastrojera & 1160.0 & 1845.7 & 1382.0 & 9.0 & 28.5 & 16.6 & $0.52^{* *}$ & 2.1 \\
\hline Terreno Arado & 2046.7 & 746.6 & 1633.2 & 10.6 & 29.0 & 21.7 & $0.64^{\star \star}$ & 2.8 \\
\hline $\begin{array}{l}\text { Rast }+ \text { T arado } \\
+ \text { Barbecho }\end{array}$ & 4070.3 & 3829.9 & 3825.3 & 24.8 & 16.8 & 8.3 & $0.78^{\star \star}$ & 4.6 \\
\hline
\end{tabular}

Cuadro II. Estimación de las superficies ocupadas por los principales usos y cultivos en las 3223 ha del Nuevo Regadio de Quinto (Zaragoza), Julio 1990.

\begin{tabular}{|c|c|c|c|c|c|c|c|c|}
\hline Clases & $\begin{array}{l}\text { xpansion }(y N) \\
\text { (ha) }\end{array}$ & $\begin{array}{l}\text { Clasificación (XN) } \\
\text { (pixeles } \\
\text { convertidos a ha) }\end{array}$ & \multicolumn{2}{|c|}{ Regresión $(\hat{Y N})$} & \multicolumn{2}{|c|}{$\begin{array}{c}\text { CV (\%) } \\
\text { Expansión Regresión }\end{array}$} & $r^{2}$ & $E R$ \\
\hline Maiz & 328.0 & 134.9 & 222.8 & 6.9 & 40.0 & 21.2 & $0.87^{\star \star}$ & 7.8 \\
\hline Alfalfa & 579.0 & 283.4 & 382.0 & 11.9 & 24.3 & 10.1 & $0.93^{\star \star}$ & 13.4 \\
\hline Rastrojera & 1120.2 & 1328.3 & 1449.5 & 45.0 & 14.9 & 7.1 & $0.51^{\star \star}$ & 2.6 \\
\hline Terreno Arado & 283.2 & 249.4 & 267.5 & 8.3 & 47.8 & 28.6 & $0.68^{* *}$ & 3.1 \\
\hline $\begin{array}{l}\text { Rast + T arado } \\
+ \text { Barbecho } \\
+ \text { Veg Espontáne }\end{array}$ & 1651.2 & 1896.4 & 1849.8 & 57.4 & 11.2 & 3.6 & $0.87^{\star \star}$ & 7.5 \\
\hline
\end{tabular}

Cuadro III. Estimación de las superficies ocupadas por los principales usos y cultivos en las 25746 ha del regadio de Flumen (Huesca), Julio 1990.

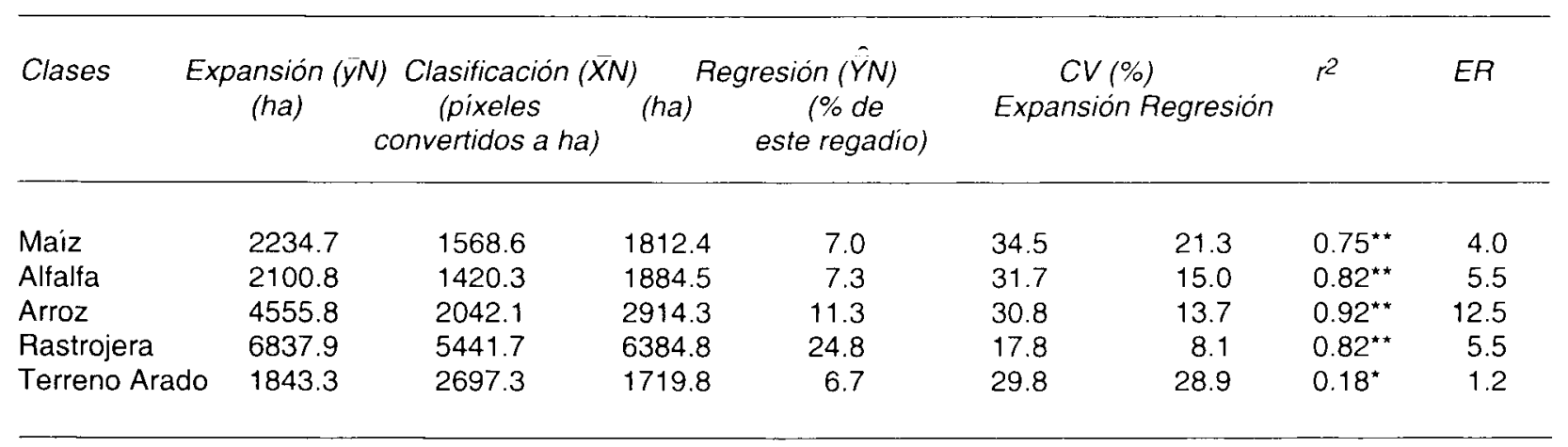

representados en Huerta Vieja y Flumen, que se delimitan en tanto que áreas dominadas por un canal y cuya ocupación es intensa y antigua. Ocurre lo contrario en el Nuevo Regadío, que es un área desértica de reciente equipamiento por aspersión.
Los Cuadros I, II y III muestran que la teledetección, mediante la aplicación del estimador por regresión, consigue en general mejorar la precisión de la estimación. Ello supone que para los cultivos, el aforo más fiable es la superficie obtenida por regresión acompañada de su coefi- 
ciente de variación. Una excepción es el arroz, por los motivos que se discuten más adelante.

Los Cuadros muestran menores coeficientes de variación para aquellos cultivos que suponen un porcentaje mayor en el regadío correspondiente. Los coeficientes de variación son elevados en las clases cuya cobertura laxa (terreno arado), o cuyo escalonamiento en la siembra (maíz del Flumen) origina una respuesta espectral heterogénea. En términos generales, la heterogeneidad espectral de cada clase se refleja en los valores del coeficiente de correlación.

\section{Maíz}

En el Nuevo Regadío de Quinto existe alta relación entre la verdad-terreno y la respuesta espectral. Los grandes tamaños de sus parcelas, necesarios para el riego mecanizado, explican que aparezcan sólo 3 ó 4 clases en unos pocos segmentos en los que se concentra la representación de la clase maiz. Ello, junto con la poca superficie del mismo $(6,9 \%)$ en el nuevo regadío hace que los coeficientes de variación sean mucho mayores que en la Huerta Vieja.

En Flumen la mejora obtenida en el coeficiente de variación al aplicar el estimador de regresión, es más modesta. Ello se debe a que el maíz sólo supone el $7,0 \%$ de la superficie de este regadio y a las diferentes respuestas espectrales consecuencia del escalonamiento de siembras. Si se desearan mejorar estos coeficientes se podría utilizar una imagen más tardía.

\section{Alfalfa}

Es aplicable lo indicado para la clase maíz en cuanto a superficies y distribución. Los coeficientes de determinación pueden considerarse satisfactorios, si bien el de la Huerta Vieja (Cuadro I) es sensiblemente menor. Esto se interpreta por la coexistencia de parcelas de alfalfa en pleno desarrollo con otras recién cortadas, que aparecían en mayor proporción en la Huerta Vieja.

\section{Arroz}

Aumenta considerablemente la precisión al aplicar el método del estimador por regresión. Las fuertes discrepancias entre el dato por regresión y el de expansión directa se deben a que las parcelas de arroz no están dispersas en el territorio, sino casi exclusivamente formando cotos arroceros en enclaves salino-sódicos. El aumento de precisión se debe a la especificidad de la firma espectral del arroz, único cultivo con inundación en estos regadios. Esto lleva a considerar como dato más fiable el obtenido por clasificación.

\section{Rastrojera}

Esta clase corresponde a cereales de invierno en regadio ya cosechados en el momento del trabajo de campo, y a parcelas aún no labradas. En Flumen la firma espectral de esta cubierta proporciona un alto coeficiente de determinación. Ello, unido a la gran importancia relativa de este uso, explica el bajo coeficiente de variación.

El el Nuevo Regadio, esta clase pese a su bajo $r^{2}$ presenta un coeficiente de variación mucho menor que el de las otras clases. Ello se atribuye al elevado porcentaje que representa en este regadio. En la Huerta Vieja, el porcentaje de la clase es mucho menor. Asi se explica que con un valor de $\mathrm{r}^{2}$ similar al del nuevo Regadío, la estimación sea peor.

\section{Terreno Arado}

En Flumen no se producen mejoras apreciables de los resultados con el empleo del proceso de imagen. La razón principal es la gran irregularidad de las respuestas espectrales de este uso, que incluye : laboreos sobre distintos tipos de suelos, tierras de labor abandonadas y áreas salinas desnudas o con vegetación halófila. Es difícil establecer la relación existente entre el concepto "terreno arado" definido en campo y su respuesta espectral.

Esto se refleja en un bajo coeficiente de determinación que no alcanza el nivel de significación exigido. Se considera más fiable la estimación por expansión.

La heterogeneidad de respuestas denota la existencia de mucha información dentro de la clase espectral. El análisis de esta información incorporando datos de la cartografia de suelos, en especial de la salinidad, para crear nuevas clases temáticas será abordado en próximos trabajos. 
En el Nuevo Regadio y la Huerta Vieja la variabilidad de la respuesta no es tan acusada, lo que se relaciona con la menor extensión y la distribución en pequeños rodales de los suelos afectados por salinidad.

\section{Sin cultivo}

Se aprecia una mejoria sustancial al ir fusionando las clases $\sin$ cultivo y con cubierta vegetal escasa o nula en el momento de paso del satélite. La superficie total aumenta y la diferente respuesta espectral de esta nueva clase frente a la de los cultivos hace que se consiga una buena clasificación. Los coeficientes de variación y la eficiencia aumentan.

\section{CONCLUSIONES}

Un método de inventario de cultivos, ya clásico para grandes superficies, se ha verificado en la estadistica de tres pequeños regadios. Se confirma así la validez de esta herramienta para la gestión de polígonos de riego.

El método de los segmentos ha permitido, mediante una única imagen Landsat, aforar las superficies de maíz, alfalfa y arroz en tres regadíos de diferentes características y tamaños $\left(257,154\right.$ y $\left.32 \mathrm{~km}^{2}\right)$. Para estos cultivos, que suponen el mayor consumo de agua, las cifras de aforo de superficies se acompañan de los coeficientes de variación, pudiéndose obtener los demás estadísticos.

En los dos regadios mayores, utilizando tasas de muestreo del $2.5 \%$ y $3.2 \%$, el estimador por regresión consigue disminuir sensiblemente los coeficientes de variación respecto a los obtenidos en la expansión directa.

Cultivos como girasol, frutales y hortícolas, dispersos y poco representados en estos regadíos y con firmas espectrales irregulares, no son aforables con este método.

Los valores de la eficiencia relativa $(E R)$ indican, para todos los cultivos y usos, que la superficie a muestrear para conseguir un determinado coeficiente de variación disminuye al aplicar la clasificación multiespectral. Desde el punto de vista de los costos de las operaciones estadísticas, los servicios correspondientes deberán valorar no sólo la mejora de la precisión y rapidez, sino también las posibilidades de localización cartográfica de los cultivos.

\section{REFERENCIAS}

Battese GE, Fuller WA (1988) Prediction of county crop areas using survey and satellite data. Proc Sect Surv Res Methods, Am Stat Assoc

Battese GE, Harter RM, Fuller WA (1988) An error components model for prediction of county crop areas using survey and satellite data. J Am Stat Assoc 83, 28-36

Casterad MA (1990) Utilización de datos de satélite para el aforo de superficies de cultivos en el centro del valle del Ebro. Trab de Invest, UPC-ETSIA, Lérida, $130 p$

CITA (1987) Remote Sensing in Agriculture. Crop Acreage Estimate and Crop Production Forecast "From Feasibility to Implementation". Consorzio Italiano per il Telerilevamento in Agricultura (CITA), Ministerio de Agricultura y Bosques de Italia, $100 \mathrm{p}$

Cochran WG (1977) Sampling Techniques. Wiley, NY, 3rd edn

Cotter J, Nealon J (1987) Area Frame Design for Agricultural Surveys. USDA, Nat Agric Stat Serv, $67 \mathrm{p}$

Delince $J(1988)$ The test site networks of the agricultural project. In: Proc 8th Earsel Symp Alpine and Mediterranean Areas: A Challenge for Remote Sensing. Capri (Nápoles) Italia, 17-20 de mayo de 1988

Ford B, Bond D, Carter N (1983) Research into small area estimation at the US Department of Agriculture. Proc Sect Surv Res Methods, Am Stat Assoc, 232-236

Gallego J (1990) El proyecto de teledetección aplicada a las estadisticas agrarias de la CEE : estimación de superficies y rendimientos de cultivos anuales. Semin sobre teledetección aplicada a la agricultura, Colegio Oficial de Ingenieros Agrónomos, Madrid

Germain MF, Julien C (1988) L'application de la télédétection à l'estimation de superficies agricoles. Can J Stat 16, 9-23

Gil C (1991) Aforo de superficies de cultivos en la zona del Flumen (Huesca) mediante teledetección multiespectral. Trabajo fin de carrera, Esc Univ Poltécnica de La Almunia, Zaragoza

González F, López S, Cuevas JM (1991) Comparing two methodologies for crop area estimation in Spain using Landsat TM images and groundgathered data. Remote Sens Environ 35, 29-35

Hanuschak G, Sigman $R$, Craig M, Ozga M, Luebbe R, Cook P, Kleweno D, Miller C (1979) Obtaining Timely Crop Area Estimates Using GroundGathered and Landsat Data. USDA, Econ, Stat Coop Serv, Tech Bull 1609

Hanuschak G, Allen R, Wington W (1982) Integration of Landsat data into the crops estimation program of USA's statistical reporting service. Machine processing of remotely sensed data processing. Purdue Univ, West Lafayette, IN 
Herrero $J(1987)$ Tendencias de salinidad en suelos del sistema de riegos Monegros-Flumen. 7e Conf sobre Hidrologia General y Aplicada, SMAGUA. Zaragoza, 411-427

Herrero J, Aragüés R (1988) Suelos afectados por salinidad en Aragón. Surcos Aragón 9, 5-8

Herrero J, Bercero A (1991) La salinidad en el nuevo regadio de Quinto (Zaragoza). Suelo Planta 1, 585602

Hultine FL, Harville DA (1991) Some Bayesian and non Bayesian procedures for the analysis of comparative experiments and for small-area estimation: computational aspects, frequentist properties and relationships. J Am Stat Assoc 86 (415), 557568

IAT (1989) Monitoring Agriculture with Remote Sensing Techniques. Flash Tele-Agri-News. CEE, CCR, Institut des Applications de la Télédétection (IAT), Ispra, Spec Publ n 1, 8934, $20 p$

Meyer-Roux J (1981) Cartographie et statistique en télédétection. Cah Stat Agric SCEES 6/6, 9-14

Meyer-Roux J (1990) Remote sensing and statistical systems, area sampling, stratification regression estimates, results, comparison of the different techniques. In: Euro-Courses: Application of Remote Sensing to Agricultural Statistics, Crop Inventories and Area Frame Sampling. Comisión de los Communidades Europeas, Consejo de Investigaciones colectivo, Ispra-Italia, RSA 90/2
Meyer-Roux J, Fournier PH, Touzelet M (1983) The remote sensing program of the French Agricultural Statistical Service. In: 17th Int Symp Remote Sensing Environ. Ann Arbor, MI, $12 \mathrm{p}$

Rodríguez R, Herrero J, Porta J (1989) Suelos de regadío con drenaje enterrado. XVI Reun $S o c$ Española Ciencia Suelo. Lérida, $93 \mathrm{p}$

SCEES (1986) Enquête sur l'utilisation du territoire effectuée en 1985 par la méthode des segments dans la Région lle-de-France et dans deux départements de la Région Centre. Service Central des Enquêtes et Études Statistiques (SCEES). Ministère de l'agriculture, France, 13, 63 $p$

SERN (1990) El uso de las imágenes de satélite en el análisis de la distribución espacial de cultivos. Aplicación metodológica en el Bajo Guadalquivir. Serv Eval Recursos Naturales (SERN), Agencia de Medio Ambiente, Junta de Andalucia, Sevilla, $131 \mathrm{p}$

TCSA (1987) Estudio de las aplicaciones de los satélites de recursos naturales en Navarra en la campaña 87 (Peralta). Trabajos Catastrales SA, Pamplona, $122 \mathrm{p}$

TCSA (1990) Informe de estadística 1990, comarcas agraria VI y VII. Método marco de áreas y estimación por regresión. Trabajos Catastrales SA, Pamplona, $248 \mathrm{p}$

Touzelet M (1983) Enquête sur l'utilisation du sol par la mèthode des segments, Ardèche 1982. Cah Stat Agric SCEES, 2/6, 9-16 